Quim. Nova, Vol. 35, No. 7, 1348-1354, 2012

\title{
MUDANÇAS QUÍMICAS E FITODISPONIBILIDADE DE ZINCO ESTIMADA POR MÉTODO ISOTÓPICO, EM SOLO TRATADO COM LODO DE ESGOTO
}

\author{
Felipe Carlos Alvarez Villanueva*, Antonio Enedi Boaretto, Lucia Pittol Firme, Takashi Muraoka, Virgilio Franco do \\ Nascimento Filho e Cassio Hamilton Abreu Junior \\ Centro de Energia Nuclear na Agricultura, Universidade de São Paulo, CP 96, 13400-970 Piracicaba - SP, Brasil
}

Recebido em 15/9/11; aceito em 25/2/12; publicado na web em 15/6/12

\begin{abstract}
CHEMICAL CHANGES AND ZINC PHYTOAVAILABILITY IN SEWAGE SLUDGE-AMENDED SOIL ESTIMATED BY THE ISOTOPIC METHOD. Zn availability in Red Latossol (Rhodic Ferralsol) of different pH amended with different rates of sewage sludge was studied by the isotopic ${ }^{65} \mathrm{Zn} L$ value method. Soil chemical properties were found to be altered by SS addition. Zn concentration and Zn derived from SS (ZnpfSS) in plant, and Zn phytoavailability ( $L$ value), were increased with increasing SS rates. The linear correlation coefficient of plant Zn with SS rates and with $L$ value was significant at $1 \%$ probability. The $L$ value proved an efficient method for predicting $\mathrm{Zn}$ phytoavailability in sewage sludge-amended soil with different $\mathrm{pH}$ under the soil conditions studied.
\end{abstract}

Keywords: $L$ value; ${ }^{65} \mathrm{Zn}$; soil chemical properties.

\section{INTRODUÇÃO}

O tratamento de esgoto urbano produz o resíduo sólido chamado lodo de esgoto (LE), que, por sua riqueza em material orgânico (MO) e nutrientes, é um produto de interesse agrícola utilizado como fonte de nitrogênio e fósforo para as plantas. ${ }^{1,2}$ Quando as águas de esgoto domiciliares são também compostas por águas provenientes de área industrial, o lodo é mais rico em elementos potencialmente tóxicos (EPT), em particular o Zn, elemento essencial às plantas e animais, por estar presente em maior concentração no LE.,13-7

O uso de LE na agricultura é restringido em normas específicas do Conselho Nacional do Meio Ambiente (CONAMA), a fim de se evitar a transferência de EPT para a cadeia alimentar. ${ }^{8}$ A aplicação repetida de lodo de esgoto para melhorar a estrutura do solo, os níveis de $\mathrm{MO}$ e de nutrientes no solo e o estado nutricional das plantas pode elevar os níveis de zinco no decorrer dos anos, o que requer atenção especial em relação ao ambiente. ${ }^{9} \mathrm{O}$ acúmulo de $\mathrm{Zn}$ em solo tratado com LE é preocupante, devido à toxicidade por este elemento ser mais frequente que com outros EPT's. ${ }^{10}$ As maiores preocupações, com a toxicidade por EPT's, dizem respeito à redução da produtividade agrícola, devido aos efeitos fitotóxicos para as plantas, à entrada na cadeia alimentar com efeitos fitotóxicos para animais e seres humanos, alteração da atividade microbiana e contaminação de recursos hídricos. ${ }^{6,11,12}$

A fitodisponibilidade do $\mathrm{Zn}$ no solo é controlada pelo teor total e, fundamentalmente, pelas formas químicas, que dependem de condições químicas, químico-físicas e físicas do solo, como $\mathrm{pH}$, teor de nutrientes, capacidade de troca iônica, condutividade elétrica, potencial óxido-redutor, MO, textura, estrutura, densidade, porosidade, permeabilidade, fluxo de água, ar e temperatura. ${ }^{13,14}$

A composição e as características dos LE variam em função da origem da água de esgoto e da tecnologia utilizada em sua obtenção. ${ }^{1,3}$ Por outro lado, as propriedades químicas e químico-físicas dos solos são alteradas pela incorporação de LE e, consequentemente, podem alterar a disponibilidade e a fitodisponibilidade de nutrientes e EPT., ${ }^{4} 14$

Os procedimentos convencionais utilizados na avaliação da disponibilidade de nutrientes ou EPT no solo são baseados na utilização

*e-mail: falvarez950@yahoo.com de soluções extratoras ácidas, salinas ou de substâncias formadoras de complexos. ${ }^{13-15}$ A fitodisponibilidade é estimada pela correlação da quantidade de elemento extraída pela solução extratora com aquela absorvida pelas plantas. Porém, de acordo com as características do solo, tipo de cultura e espécie química extraída, nem sempre esses procedimentos têm sido eficientes para predizer a fitodisponibilidade de nutrientes e EPT's. ${ }^{15-18}$

Os procedimentos estabelecidos para a extração química de EPT do solo, cujos dados são correlacionados com os EPT's extraídos pelas plantas, poderiam subestimar o cenário real dos EPT's no solo. ${ }^{18}$ A seleção de um extrator químico para avaliar a disponibilidade de EPT em solo é uma tarefa complexa, pois um extrator ideal deveria ter um caráter multielementar e, ao mesmo tempo, uma elevada capacidade de diagnosticar a disponibilidade desses elementos para várias espécies vegetais e em diferentes tipos de solos. ${ }^{19}$

A fitodisponibilidade de nutrientes ou de EPT's no solo para as plantas é governada pelos seguintes fatores: diferentes interações das propriedades físicas, químicas e biológicas do solo, condições ambientais e mecanismos fisiológicos que interferem na nutrição das plantas. ${ }^{20} \mathrm{~A}$ absorção de elementos pelas raízes das plantas é um processo físico-químico em relação ao solo e físico-químico-fisiológico em relação ao sistema solo-planta. Já a determinação da disponibilidade dos elementos no solo, realizada com extratores químicos, é de caráter químico-físico, portanto, a causa da baixa eficiência na previsão da fitodisponibilidade poderia ser que esses métodos não simulem as reações que ocorrem nas raízes das plantas, ${ }^{17}$ ou seja, no processo fisiológico da planta.

A fitodisponibilidade de um nutriente ou de um EPT, expressada pela própria planta, pode ser determinada por meio de técnicas isotópicas, com os métodos direto ou indireto, que utilizam traçadores isotópicos, radioativos ou estáveis. ${ }^{21,22} \mathrm{O}$ método direto é empregado quando se dispõe de fertilizantes marcados com o isótopo radioativo, ou enriquecidos com isótopo estável. Neste método é utilizada a relação das atividades específicas (radioisótopo) ou excesso de átomos (isótopo estável) do isótopo contido nas plantas e da fonte fertilizante que foi adicionada ao solo, para identificar o nutriente ou EPT de interesse. ${ }^{22} \mathrm{O}$ método indireto é utilizado para avaliar fontes portadoras de nutrientes ou EPT que não podem ser marcadas ou enriquecidas com um isótopo sem modificar a sua natureza como, 
por exemplo, rochas fosfóricas, resíduos industriais ou fertilizantes orgânicos. Neste método, são empregados dois tratamentos, um de referência (testemunha) onde é aplicado o isótopo, mas não a fonte de nutriente, e outro onde são adicionados o isótopo e a fonte de nutriente. Ressalta-se que o traçador isotópico pode ser adicionado antes ou depois de adicionada a fonte portadora dos nutrientes ou EPT ao solo. Neste caso, é utilizada a relação das atividades específicas ou excesso de átomos do isótopo, nas plantas crescidas no solo que recebeu a fonte de nutriente, e das plantas crescidas no solo testemunha, para avaliar a fitodisponibilidade dos elementos de interesse. ${ }^{22}$

A grande vantagem do método isotópico, quando comparado aos extratores químicos, é que não modifica o equilíbrio químico no solo, pois são as plantas que atuam como extratoras (parâmetro fisiológico) da fração daquele elemento no solo, ou da fonte do elemento adicionada (fertilizante), que participa no intercâmbio com o isótopo (radioativo ou estável) adicionado (parâmetro químico-físico). O uso de plantas, combinado com o método isotópico, para determinar a fitodisponibilidade, representada pelo valor $L,{ }^{21}$ permite afirmar que este parâmetro é condicionado pela interação das condições físico-químicas do solo e do processo fisiológico das raízes da planta na absorção dos elementos, refletindo na fitodisponibilidade real do nutriente ou EPT. Por outro lado, o método isotópico também permite conhecer qual a origem do elemento absorvido pelas plantas, se é daquele natural contido no solo ou na fonte adicionada ao solo, LE por exemplo. ${ }^{22}$

A dinâmica dos EPT's em solos brasileiros, principalmente sua disponibilidade e fitodisponibilidade em solos agrícolas, tem sido tema de várias pesquisas, mas, dada a dimensão continental do Brasil, grande diversidade de solo, clima e LE, necessita ainda de mais estudos. O conhecimento da disponibilidade e fitodisponibilidade do EPT em solos agrícolas, principalmente os que recebem LE, é importante e pode oferecer informações valiosas para regulamentação do uso de LE na agricultura, sem riscos de impactos negativos ao ambiente e à cadeia alimentar.

O presente trabalho teve como objetivo determinar a fitodisponibilidade do Zn e o acumulado por plantas de milho (Zea mais L.) proveniente do solo (Znpps) e do LE (ZnppLE), em um Latossolo Vermelho (Rhodic Ferralsol) ${ }^{23}$ com diferentes valores de $\mathrm{pH}$, tratado com LE, mediante uso do método isotópico do valor $L, \operatorname{com}{ }^{65} \mathrm{Zn}$.

\section{PARTE EXPERIMENTAL}

\section{Caracterização do solo e do lodo}

O trabalho foi realizado em casa de vegetação, do Laboratório de Nutrição Mineral de Plantas do CENA/USP, Piracicaba, SP, utilizando amostra de solo coletada da camada superficial de $0-20 \mathrm{~cm}$ de profundidade de um Latossolo Vermelho. Essa amostra foi seca ao ar, passada por peneira com malha de $5 \mathrm{~mm}$ e homogeneizada. Uma amostra de $100 \mathrm{~g}$ da terra foi retirada e passada por peneira de $2 \mathrm{~mm}$ para a realização das análises granulométricas pelo método do densímetro ${ }^{24}$ e químicas, para fins de fertilidade, de acordo com método descrito em Raij et al., ${ }^{25}$ e análises dos metais $\mathrm{Zn}, \mathrm{Cd}, \mathrm{Cu}, \mathrm{Ni}$ e Pb pelo método 3050b, do manual SW-846, da U.S. EPA ${ }^{26}$ (Tabela 1) e analisados por espectrometria de emissão com fonte de plasma induzida. As concentrações encontradas, desses elementos, na amostra de solo estavam abaixo dos valores de referência para qualidade de prevenção, estabelecidos na resolução № 420 do CONAMA. ${ }^{27}$

O lodo de esgoto utilizado, denominado "Jundfertil" (Tabela 1) foi obtido na Companhia de Saneamento da cidade de Jundiaí, SP. Este material foi seco, moído e passado em peneira com malha de 2 mm e foram determinados o $\mathrm{pH}$ e a composição elementar, base seca, pelos métodos $9045 \mathrm{~b}$ e $3050 \mathrm{~b}$, respectivamente, do manual SW-846
Tabela 1. Características do solo e do lodo, valores de referência de alguns elementos para qualidade de solo (5) e concentrações máximas permitidas no LE (7)

\begin{tabular}{|c|c|c|c|c|c|c|}
\hline & & Solo & (5) & & $(6)$ & (7) \\
\hline $\mathrm{pH}$ & 4,3 & $\mathrm{CaCl}_{2}$ & & 3,7 & $\mathrm{H}_{2} \mathrm{O}$ & \\
\hline MO & 39,0 & $\mathrm{~g} \mathrm{~kg}^{-1}$ & & 348 & $\mathrm{~g} \mathrm{~kg}^{-1}$ & \\
\hline $\mathrm{P}$ & 11,0 & (1) $\mathrm{g} \mathrm{dm}^{-3}$ & & 5,5 & $\mathrm{~g} \mathrm{~kg}^{-1}$ & \\
\hline K & 0,9 & ${ }^{(1)} \mathrm{mmol}_{\mathrm{c}} \mathrm{dm}^{-3}$ & & 1,0 & $\mathrm{~g} \mathrm{~kg}^{-1}$ & \\
\hline $\mathrm{Ca}$ & 12,0 & ${ }^{(1)} \mathrm{mmol}_{\mathrm{c}} \mathrm{dm}^{-3}$ & & 10,8 & $\mathrm{~g} \mathrm{~kg}^{-1}$ & \\
\hline $\mathrm{Mg}$ & 10,0 & ${ }^{(1)} \mathrm{mmol}_{\mathrm{c}} \mathrm{dm}^{-3}$ & & 1,7 & $\mathrm{~g} \mathrm{~kg}^{-1}$ & \\
\hline $\mathrm{H}^{+}+\mathrm{Al}^{3+}$ & 52,0 & $\mathrm{mmol}_{\mathrm{c}} \mathrm{dm}^{-3}$ & & - & - & \\
\hline S & 22,9 & ${ }^{(2)} \mathrm{mmol}_{\mathrm{c}} \mathrm{dm}^{-3}$ & & - & - & \\
\hline CTC & 74,9 & ${ }^{(3)} \mathrm{mmol}_{\mathrm{c}} \mathrm{dm}^{-3}$ & & - & - & \\
\hline V & 30,6 & (4) $\%$ & & - & - & \\
\hline${ }^{(5)} \mathrm{Zn}$ & 26,1 & $\mathrm{mg} \mathrm{kg}^{-1}$ & 300 & 1,96 & $\mathrm{~g} \mathrm{~kg}^{-1}$ & 2,8 \\
\hline${ }^{(5)} \mathrm{Cu}$ & 16,6 & $\mathrm{mg} \mathrm{kg}{ }^{-1}$ & 60 & 471,8 & $m g \mathrm{~kg}^{-1}$ & 1500 \\
\hline${ }^{(5)} \mathrm{Cd}$ & 0,33 & $\mathrm{mg} \mathrm{kg}^{-1}$ & 1,3 & 16,98 & $\mathrm{mg} \mathrm{kg}^{-1}$ & 39 \\
\hline${ }^{(5)} \mathrm{Mn}$ & 98,4 & $\mathrm{mg} \mathrm{kg}^{-1}$ & - & 925,5 & $\mathrm{mg} \mathrm{kg}{ }^{-1}$ & - \\
\hline${ }^{(5)} \mathrm{Ni}$ & 0,04 & $\mathrm{mg} \mathrm{kg}^{-1}$ & 30 & 92,0 & $\mathrm{mg} \mathrm{kg}{ }^{-1}$ & 420 \\
\hline${ }^{(5)} \mathrm{Pb}$ & 0,62 & $\mathrm{mg} \mathrm{kg}^{-1}$ & 72 & 174,1 & $\mathrm{mg} \mathrm{kg}^{-1}$ & 300 \\
\hline Areia & 510 & $\mathrm{~g} \mathrm{~kg}^{-1}$ & & - & - & \\
\hline Silte & 40 & $\mathrm{~g} \mathrm{~kg}^{-1}$ & & - & - & \\
\hline Argila & 450 & $\mathrm{~g} \mathrm{~kg}^{-1}$ & & - & - & \\
\hline Textura & Argilosa & & & - & - & \\
\hline
\end{tabular}

${ }^{(1)}$ Resina trocadora de íons; ${ }^{(2)}$ soma de bases; ${ }^{(3)}$ capacidade de troca catiônica; ${ }^{(4)}$ saturação por bases; ${ }^{(6)}$ método 3050b U.S.EPA (2007).

da U.S. EPA, ${ }^{26}$ e a MO determinada segundo Nelson e Sommer. ${ }^{28}$ As concentrações encontradas de $\mathrm{Zn}, \mathrm{Cd}, \mathrm{Cu}, \mathrm{Ni}$ e $\mathrm{Pb}$, na análise por espectrometria de emissão com fonte de plasma induzida, foram inferiores aos níveis máximos permitidos na resolução № 375 do CONAMA ${ }^{8}$ que regula a qualidade de LE para aplicação agrícola (Tabela 1).

\section{Tratamento do solo e LE}

Os tratamentos foram constituídos por três valores de $\mathrm{pH}$ do solo, 4,3; 5,3 e 5,9, medidos em $\mathrm{CaCl}_{2}$ 0,01 mol L ${ }^{-1},{ }^{25}$ e cinco doses de LE, equivalentes a $0,15,30,45$ e $60 \mathrm{Mg} \mathrm{ha}^{-1}$ (base seca). As doses de LE foram calculadas com o cuidado para que a maior dose não excedesse o limite máximo permitido para $\mathrm{Cd}$ no solo, cujo valor de investigação é $3 \mathrm{mg} \mathrm{kg}^{-1}$, por ser este o EPT mais limitante, de acordo com os critérios da resolução № 420 do CONAMA. ${ }^{27}$ Após a instalação do experimento, considerando-se os teores de $\mathrm{Zn}, \mathrm{Cd}$, $\mathrm{Cu}, \mathrm{Ni}$ e $\mathrm{Pb}$ como a somatória dos teores de cada EPT contidos no solo com LE, no tratamento com a maior dose de LE (60 $\left.\mathrm{Mg} \mathrm{ha}^{-1}\right)$, os teores de $\mathrm{Zn}, \mathrm{Cd}$, $\mathrm{Cu}$, $\mathrm{Ni}$ e $\mathrm{Pb}$, expressos em mg kg-1 aumentaram para $85 ; 1,75 ; 30,8 ; 12,7$ e 14,7, respectivamente, estando ainda abaixo do nível de investigação para o $\mathrm{Cd}$ e de prevenção para o Zn, Cu, Ni e $\mathrm{Pb}$, permitidos na resolução № 420 do CONAMA ${ }^{27} \mathrm{O}$ experimento foi realizado em delineamento inteiramente casualizado, em esquema fatorial 3 x 5, com três repetições, sendo as parcelas compostas por vasos de plástico de $2,5 \mathrm{dm}^{3}$.

A amostra de solo coletada, com $\mathrm{pH}\left(\mathrm{CaCl}_{2}\right)=4,3$, foi dividida em três subamostras iguais e em duas delas foi ajustado o $\mathrm{pH}\left(\mathrm{CaCl}_{2}\right)$ a valores de 5,3 e 5,9, respectivamente, mediante adição de mistura 
de carbonato de cálcio e carbonato de magnésio, de grau analítico, com relação 4:1 de Ca:Mg e incubação durante 30 dias.

Posteriormente, em vasos plásticos de $2,5 \mathrm{dm}^{3}$ recobertos internamente com sacos plástico, adicionaram-se $2 \mathrm{~kg}$ de terra seca com as respectivas doses de LE. Após homogeneização, incubaram-se as amostras de terra por mais 30 dias. De cada vaso foram retiradas amostras de terra, secas ao ar e passadas por peneira de $2 \mathrm{~mm}$, para avaliação das alterações químicas e químico-físicas provocadas pela adição de LE. As análises realizadas, seguindo os métodos descritos em Raij et al., ${ }^{25}$ foram $\mathrm{pH}$ em $\mathrm{CaCl}_{2} 0,01 \mathrm{~mol} \mathrm{~L}^{-1}$, acidez potencial $\left(\mathrm{H}^{+}+\mathrm{Al}^{3+}\right), \mathrm{MO}, \mathrm{Ca}$ e $\mathrm{Mg}$ trocáveis, e a capacidade de troca de cátions (CTC).

Após a amostragem da terra, adicionaram-se $100 \mathrm{~mL}$ de uma solução de $\mathrm{ZnSO}_{4}$ contendo $3 \mu \mathrm{g}$ de $\mathrm{Zn}$ e $148 \mathrm{kBq}(4 \mu \mathrm{Ci})$ de ${ }^{65} \mathrm{Zn}$, em cada vaso. Dois dias depois, as amostras de solo marcadas com o ${ }^{65} \mathrm{Zn}$ foram novamente homogeneizadas e incubadas por 30 dias. Todas as incubações foram realizadas mantendo-se a umidade da amostra de solo a $70 \%$ da capacidade de retenção de água, controlada com a medição da massa dos vasos e a reposição da água evaporada quando necessário.

Posteriormente procedeu-se à adubação com potássio; como a concentração desse elemento no LE era baixa (Tabela 1), todos os tratamentos receberam $150 \mathrm{mg} \mathrm{kg}^{-1}$ do elemento na forma de cloreto de potássio, de grau analítico.

\section{Instalação do experimento com planta de milho}

Em cada vaso foram semeadas 5 sementes de milho (Zea mais L.), híbrido Pioneer 30F80. Cinco dias após a germinação, foi realizado um desbaste e deixadas 2 plantas por vaso.

$\mathrm{O}$ tratamento testemunha $\left(0 \mathrm{Mg} \mathrm{ha}^{-1} \mathrm{de} \mathrm{LE}\right)$ recebeu adubo nitrogenado na dose de $50 \mathrm{mg} \mathrm{kg}^{-1}$ no momento da semeadura e $150 \mathrm{mg}$ $\mathrm{kg}^{-1}$ aos 15 dias após a germinação. Para evitar a variação do $\mathrm{pH}$ das amostras de solo pelo adubo nitrogenado, utilizou-se uma mistura de $79,85 \%$ de $\mathrm{N}$ na forma de nitrato de cálcio e 20,15\% na forma de sulfato de amônio, seguindo a recomendação de Raij et al.. ${ }^{29}$ Neste tratamento, também se adicionaram $0,5 \mathrm{mg} \mathrm{kg}^{-1} \mathrm{de} \mathrm{Cu}$ (sulfato de cobre); 0,5 mg kg-1 de B (ácido bórico); $8,1 \mathrm{mg} \mathrm{kg}^{-1}$ de Mn (sulfato de manganês) e $0,2 \mathrm{mg} \mathrm{kg}^{-1}$ de Mo (molibdato de amônio), todos na forma de reagentes de grau analítico.

Aos 40 dias após a germinação, a parte aérea das plantas (folhas e talos) foi cortada a aproximadamente $1 \mathrm{~cm}$ de altura da superfície da terra, lavada com água desionizada de condutividade $0,6 \mu \mathrm{S} \mathrm{cm}^{-1}$, seca em estufa a $65^{\circ} \mathrm{C}$, pesada e moída. Subamostras de planta foram digeridas em solução nítrica-perclórica ${ }^{30} \mathrm{e}$ realizada análise de $\mathrm{Zn}$.

\section{Métodos analíticos}

O método analítico empregado para análise dos elementos $\mathrm{Zn}$, $\mathrm{Cd}, \mathrm{Cu}, \mathrm{Ni}$ e $\mathrm{Pb}$ no solo e no LE e de $\mathrm{Zn}$ nas plantas foi a espectrometria de emissão com fonte de plasma.

Para verificação da qualidade das análises, junto com as amostras de solo, LE e planta, foram realizadas análises em triplicata de amostras certificadas, de solo (NIST 2710), de lodo (NIST 2781) e de planta (NIST 1515). A exatidão das análises realizadas nas amostras certificadas foi avaliada mediante cálculo da porcentagem de recuperação $(\mathrm{R})$ dos analitos. A recuperação é definida como a proporção da quantidade de analito obtido, por meio do método analítico, em relação à quantidade certificada na amostra. ${ }^{31} \mathrm{~A} \mathrm{R}$ é calculada pela Equação 1:

$$
R \%=\frac{C}{C_{M R C}} \times 100
$$

onde: $C$ é o valor médio da concentração do analito, obtido das $n$ análises realizadas e $C_{M R C}$, a concentração de analito certificada no material de referência. Os valores obtidos de $\mathrm{R}$ para $\mathrm{Zn}, \mathrm{Cd}, \mathrm{Cu}$, $\mathrm{Ni}$ e $\mathrm{Pb}$, no NIST SRM 2710 (solo) foram de 91,45; 90,6; 92,1; 93,4 e 92,2\%, respectivamente; no NIST SRM 2781 (lodo) foram de 90,$1 ; 93,2 ; 94,1 ; 92,5$ e 96,3\%, respectivamente; a recuperação de Zn no NIST SRM 1515 foi de 95,2\%. Considerando a impossibilidade de se obter um resultado experimental exatamente igual ao valor certificado, convencionalmente aceito como verdadeiro, ${ }^{32}$ pode-se considerar que o procedimento empregado para análise dos elementos foi adequado.

As medidas de radioatividade do ${ }^{65} \mathrm{Zn}$, em $2 \mathrm{~g}$ de amostra de planta seca, foram realizadas por espectrometria gama em analisador multicanal acoplado a detector semicondutor de Ge(Li) hiperpuro, utilizando-se o fotopico de $1115 \mathrm{keV}$ e tempo de medição de $1000 \mathrm{~s}$.

$\mathrm{O}$ valor $L$ foi calculado pela relação das atividades específicas do ${ }^{65} \mathrm{Zn}$ adicionado ao solo $\left(A_{s}\right)$ e a encontrada nas plantas $\left(A_{p}\right)$ de acordo com a Equação 2:

$$
\text { valor } L=Z n_{c} \times\left(\frac{A_{s}}{A_{p}}-1\right)
$$

onde: $Z n_{c}=3 \mu \mathrm{g} \mathrm{kg}^{-1}$ de $\mathrm{Zn}$ estável utilizado como carregador do radioisótopo ${ }^{65} \mathrm{Zn}$.

As medidas de radioatividade nas plantas foram utilizadas também para calcular as porcentagens de Zn na planta proveniente do solo (\%Znpps) e do LE (\%ZnppLE), por meio da relação das atividades específicas do ${ }^{65} \mathrm{Zn}$ nas plantas nos tratamentos com LE $\left(A_{L E}\right)$ e no tratamento referência, sem adição de LE $\left(A_{0}\right)$ :

$$
\% \text { Znpps }=\frac{A_{L E}}{A_{0}} \times 100
$$

e

$$
\% \text { ZnppLE }=100-\% \text { Znpps }
$$

Para a interpretação dos resultados, foram realizadas análises de variância e de regressão utilizando-se do programa Statistical Analysis System (SAS). ${ }^{33}$ Sempre que a análise de variância (teste F) detectou diferenças significativas entre tratamentos, realizou-se a análise da comparação múltipla das médias dos tratamentos através do teste Tukey a 5\% de probabilidade e avaliações por meio de regressões lineares simples e polinomiais.

\section{RESULTADOS E DISCUSSÃO}

A adição de LE ao solo alterou as propriedades químicas do solo, 30 dias após a incubação com o resíduo. Houve diminuição linear dos valores de $\mathrm{pH}\left(\mathrm{CaCl}_{2}\right)$ e aumento da acidez potencial $\left(\mathrm{H}^{+}\right.$ $+\mathrm{Al}^{3+}$ ), em função das doses de LE (Tabela 2). Resultado semelhante foi obtido por outros autores ${ }^{34}$ ao utilizarem doses de lodo de $\mathrm{pH}$ (em água) = 6,1 da Estação de Mangueira, Recife, PE, em solo Argissolo Vermelho-Amarelo e Espodossolo Cárbico hidromórfico de $\mathrm{pH}=$ 5,2 e 5,0, medidos em água, respectivamente. Em outros trabalhos, nos quais empregaram LE de $\mathrm{pH}$ neutro ou alcalino, provenientes de processos que utilizaram cal para higienização e estabilização do resíduo, ${ }^{3}$ foi reportado aumento no valor de $\mathrm{pH}$ do solo. ${ }^{19,35}$ Portanto, a alteração do equilíbrio ácido-base do solo é dependente da reação ácida ou alcalina do LE, segundo o tratamento de estabilização utilizado na sua obtenção, e da dose do resíduo aplicada no solo, além da textura e capacidade tamponante do solo. ${ }^{36}$ Outras causas de acidificação do solo podem ser atribuídas a reações de imobilização de nitrogênio, ou nitrificação, e à formação de ácidos orgânicos durante 
a degradação da MO por micro-organismos, ${ }^{20}$ conforme representado pelas Equações 5 a 7 :

Imobilização $\mathrm{NH}_{4}^{+}+\mathrm{R}-\mathrm{OH} \rightarrow \mathrm{R}-\mathrm{NH}_{2}+\mathrm{H}_{2} \mathrm{O}+\mathrm{H}^{+}$

Nitrificação $\mathrm{NH}_{4}^{+}+2 \mathrm{O}_{2} \rightarrow \mathrm{NO}_{3}^{-}+\mathrm{H}_{2} \mathrm{O}+2 \mathrm{H}^{+}$

Decomposição microbiana $\mathrm{C}$ orgânico $\rightarrow \mathrm{R}^{-\mathrm{COO}^{-}+\mathrm{H}^{+}}$

Em trabalho avaliando a aplicação de 0,10, 20, 30, 40 e $50 \mathrm{Mg}$ $\mathrm{ha}^{-1}$ de LE, proveniente da estação de Tratamento de águas do Ribeirão do Piracicamirim, Piracicaba, SP, os autores reportaram diminuição de $\mathrm{pH}$ do solo devido à mineralização da MO aportada pelo LE. ${ }^{37}$

A acidez potencial $\left(\mathrm{H}^{+}+\mathrm{Al}^{3+}\right)$ no solo aumentou linearmente com as doses de LE. O maior valor de acidez potencial ocorreu na amostra de solo com $\mathrm{pH}=4,3$, fato favorecido pela própria acidez do solo mais a aportada pelo LE (Tabela 2); entretanto, nas amostras de solo com $\mathrm{pH}=5,3$ e 5,9 as quantidades de $\left(\mathrm{H}^{+}+\mathrm{Al}^{3+}\right)$ foram menores, devido à ação neutralizante da acidez aportada pelo LE nesses solos.

$\mathrm{O}$ teor de MO nas amostras de solo aumentou linearmente com as doses de LE. Os aumentos, para a maior dose de LE e em relação ao tratamento de referência sem LE, foram de 33, 27 e 48\%, nas amostras de solo com $\mathrm{pH}=4,3 ; 5,3$ e 5,9, respectivamente, (Tabela 2). $\mathrm{O}$ aporte de $\mathrm{MO}$, conjuntamente com a manutenção de $\mathrm{pH}$ adequado para as plantas $(5,5$ a 6,5$)$, é especialmente importante para minimizar a disponibilidade de EPT em solo. ${ }^{13,14} \mathrm{~A}$ MO diminui a atividade dos íons dos EPT por complexação ou por mecanismos de adsorção e troca de íons, enquanto o aumento de $\mathrm{pH}$ diminui a atividade por formação de compostos de baixa solubilidade ou aumento da adsorção na fase sólida do solo.

$\mathrm{O}$ teor de $\mathrm{K}$, mesmo que tenha aumentado de $0,96 \mathrm{mmol}_{\mathrm{c}} \mathrm{dm}^{-3}$, sem aplicação de LE, até $1,33 \mathrm{mmol}_{\mathrm{c}} \mathrm{dm}^{-3}$, na maior dose de LE, permaneceu na faixa de teor considerado baixa, ${ }^{25}$ devido à baixa concentração do elemento no LE, fato que determinou a necessidade de aplicar adubo potássico na semeadura.

Os teores de $\mathrm{Ca}$ e $\mathrm{Mg}$ trocáveis aumentaram linearmente com as doses de LE (Tabela 2). Segundo os critérios estabelecidos para fins de fertilidade dos solos, ${ }^{25}$ o teor de Ca trocável era baixo $(<20$ $\left.\mathrm{mmol}_{\mathrm{c}} \mathrm{dm}^{-3}\right)$, médio $\left(20 \mathrm{a} 40 \mathrm{mmol}_{\mathrm{c}} \mathrm{dm}^{-3}\right)$ e alto $\left(>40 \mathrm{mmol}_{\mathrm{c}} \mathrm{dm}^{-3}\right)$ nas terras de $\mathrm{pH}=4,3 ; 5,3$ e 5,9, respectivamente, enquanto que o teor de $\mathrm{Mg}$ era alto $\left(>8 \mathrm{mmol}_{\mathrm{c}} \mathrm{dm}^{-3}\right)$ nas três amostras de solo. Trinta dias após adição do LE, o teor de Ca passou de baixo para médio na amostra de solo com $\mathrm{pH}=4,3$ e de médio para alto na terra com $\mathrm{pH}$ 5,3 , permanecendo na categoria de teor alto na terra de $\mathrm{pH} 5,9$. Porém, o Mg trocável continuou na faixa de teor alto (Tabela 2).

A CTC aumentou linearmente com as doses de LE e, em relação ao $\mathrm{pH}$ da terra, $\mathrm{o}$ aumento foi na ordem de $\mathrm{pH}=5,9>\mathrm{pH}=5,3>\mathrm{pH}$ $=4,3$ (Tabela 2). Todavia, como observado em outros trabalhos, ${ }^{19,34} \mathrm{a}$ determinação da CTC do solo por meio da soma de bases trocáveis e acidez potencial, conforme realizada no presente trabalho, pode ocasionar a superestimação desse índice por causa dos aportes de $\mathrm{Ca}$ e $\mathrm{Mg}$ do lodo e da calagem realizada.

Tanto a calagem realizada na terra para ajuste do $\mathrm{pH}$ a valores aproximados de 5,0 e 6,0, como as doses de LE adicionadas, contribuíram para as alterações químicas observadas nas terras dos diferentes tratamentos.

O rendimento de matéria seca da parte aérea das plantas de milho aumentou com aplicação de LE, mas em geral foi baixa (Tabela 3), devido à baixa disponibilidade de nutrientes no solo, principalmente de fósforo. $\mathrm{O}$ teor de $\mathrm{P}$ disponível no solo apresentou-se baixo, ${ }^{25}$ entre 7 e $15 \mathrm{mg} \mathrm{dm}^{-3}$. Entretanto, a aplicação de 15, 30 e $45 \mathrm{Mg} \mathrm{ha}^{-1}$ de lodo aumentou o teor de $\mathrm{P}$ para teores médios de 22,32 e $38 \mathrm{mg}$ $\mathrm{dm}^{-3}$, respectivamente, enquanto que na dose de $60 \mathrm{Mg} \mathrm{ha}^{-1}$ aumentou para $47 \mathrm{mg} \mathrm{dm}^{-3}$, teor considerado alto. ${ }^{25} \mathrm{~A}$ acidez do solo na maioria dos tratamentos, com valores de $\mathrm{pH}<5,5$, que é prejudicial
Tabela 2. Variações de $\mathrm{pH},\left(\mathrm{H}^{+}+\mathrm{Al}^{3+}\right), \mathrm{MO}, \mathrm{Ca}$ e Mg trocáveis e CTC

\begin{tabular}{|c|c|c|c|}
\hline \multirow{3}{*}{$\begin{array}{c}\text { Doses de LE } \\
\mathrm{Mg} \mathrm{ha}^{-1}\end{array}$} & \multicolumn{3}{|c|}{ pH inicial da amostra do solo } \\
\hline & 4,3 & 5,3 & 5,9 \\
\hline & \multicolumn{3}{|c|}{$\mathrm{pH}\left(\mathrm{CaCl}_{2} 0,01 \mathrm{~mol} \mathrm{~L}^{-1}\right)$} \\
\hline 0 & $4,3 \pm 0,1$ & $5,3 \pm 0,2$ & $5,9 \pm 0,1$ \\
\hline 15 & $4,2 \pm 0,1$ & $5,1 \pm 0,1$ & $5,7 \pm 0,1$ \\
\hline 30 & $4,1 \pm 0,1$ & $4,9 \pm 0,1$ & $5,6 \pm 0,2$ \\
\hline 45 & $3,9 \pm 0,2$ & $4,8 \pm 0,1$ & $5,3 \pm 0,1$ \\
\hline 60 & $4,3 \pm 0,2$ & $4,5 \pm 0,1$ & $5,2 \pm 0,2$ \\
\hline \multirow[t]{2}{*}{ Modelo } & $\begin{array}{c}y=-0,004 x+4,2 \\
R^{2}=0,229 n s\end{array}$ & $\begin{array}{c}\mathrm{y}=-0,012 \mathrm{x}+5,2 \\
\mathrm{R}^{2}=0,984^{* *}\end{array}$ & $\begin{array}{c}\mathrm{y}=-0,01 \mathrm{x}+5,8 \\
\mathrm{R}^{2}=0,973^{* *}\end{array}$ \\
\hline & \multicolumn{3}{|c|}{$\mathrm{H}^{+}+\mathrm{Al}^{3+}\left(\mathrm{mmol}_{\mathrm{c}} \mathrm{dm}^{-3}\right)$} \\
\hline 0 & $46 \pm 5$ & $30 \pm 1$ & $21 \pm 1$ \\
\hline 15 & $51 \pm 6$ & $31 \pm 3$ & $24 \pm 3$ \\
\hline 30 & $56 \pm 4$ & $35 \pm 5$ & $26 \pm 3$ \\
\hline 45 & $61 \pm 5$ & $40 \pm 6$ & $28 \pm 4$ \\
\hline 60 & $68 \pm 2$ & $48 \pm 5$ & $30 \pm 3$ \\
\hline \multirow[t]{2}{*}{ Modelo } & $\begin{array}{c}\mathrm{y}=0,37 \mathrm{x}+45 \\
\mathrm{R}^{2}=0,995^{* *}\end{array}$ & $\begin{array}{c}\mathrm{y}=0,29 \mathrm{x}+28 \\
\mathrm{R}^{2}=0,914^{* *}\end{array}$ & $\begin{array}{c}\mathrm{y}=0,14 \mathrm{x}+22 \\
\mathrm{R}^{2}=0,993^{* *}\end{array}$ \\
\hline & \multicolumn{3}{|c|}{ Matéria orgânica $\left(\mathrm{g} \mathrm{kg}^{-1}\right)(\mathrm{MO})$} \\
\hline 0 & $31 \pm 1$ & $34 \pm 2$ & $30 \pm 3$ \\
\hline 15 & $36 \pm 2$ & $35 \pm 3$ & $33 \pm 2$ \\
\hline 30 & $38 \pm 3$ & $39 \pm 3$ & $37 \pm 1$ \\
\hline 45 & $40 \pm 4$ & $40 \pm 4$ & $41 \pm 2$ \\
\hline 60 & $42 \pm 3$ & $43 \pm 3$ & $46 \pm 5$ \\
\hline \multirow[t]{2}{*}{ Modelo } & $\begin{array}{c}y=0,16 x+32 \\
R^{2}=0,973 * *\end{array}$ & $\begin{array}{c}y=0,16 x+33 \\
R^{2}=0,962^{* *}\end{array}$ & $\begin{array}{c}y=0,25 x+30 \\
R^{2}=0,989 * *\end{array}$ \\
\hline & \multicolumn{3}{|c|}{ Ca trocável $\left(\mathrm{mmol}_{\mathrm{c}} \mathrm{dm}^{-3}\right)$} \\
\hline 0 & $12,7 \pm 0,7$ & $34 \pm 2$ & $52 \pm 4$ \\
\hline 15 & $18,8 \pm 0,9$ & $38 \pm 2$ & $57 \pm 4$ \\
\hline 30 & $24 \pm 1$ & $42 \pm 2$ & $66 \pm 4$ \\
\hline 45 & $29 \pm 1$ & $46 \pm 3$ & $69 \pm 4$ \\
\hline 60 & $35 \pm 2$ & $56 \pm 3$ & $82 \pm 4$ \\
\hline \multirow[t]{2}{*}{ Modelo } & $\begin{array}{c}\mathrm{y}=0,37 \mathrm{x}+12,9 \\
\mathrm{R}^{2}=0,999 * *\end{array}$ & $\begin{array}{c}y=0,36 x+32 \\
R^{2}=0,951 * *\end{array}$ & $\begin{array}{c}y=0,48 x+51 \\
R^{2}=0,956^{* *}\end{array}$ \\
\hline & \multicolumn{3}{|c|}{$\mathrm{Mg}$ trocável $\left(\mathrm{mmol}_{\mathrm{c}} \mathrm{dm}^{-3}\right)$} \\
\hline 0 & $10,9 \pm 0,9$ & $19 \pm 1$ & $26 \pm 1$ \\
\hline 15 & $12 \pm 1$ & $22 \pm 1$ & $29 \pm 2$ \\
\hline 30 & $13 \pm 1$ & $22 \pm 1$ & $31 \pm 2$ \\
\hline 45 & $14,2 \pm 0,9$ & $23 \pm 1$ & $32 \pm 2$ \\
\hline 60 & $16 \pm 1$ & $24 \pm 1$ & $34 \pm 2$ \\
\hline \multirow[t]{2}{*}{ Modelo } & $\begin{array}{c}y=0,08 x+10 \\
R^{2}=0,982 * *\end{array}$ & $\begin{array}{c}y=0,07 x+19 \\
R^{2}=0,911^{* *}\end{array}$ & $\begin{array}{c}y=0,12 x+27 \\
R^{2}=0,982 * *\end{array}$ \\
\hline & \multicolumn{3}{|c|}{ Capacidade de troca catiônica $\left(\mathrm{mmol}_{\mathrm{c}} \mathrm{dm}^{-3}\right)(\mathrm{CTC})$} \\
\hline 0 & $71 \pm 6$ & $85,5 \pm 0,2$ & $101,1 \pm 0,7$ \\
\hline 15 & $88 \pm 11$ & $97 \pm 11$ & $111 \pm 3$ \\
\hline 30 & $98 \pm 7$ & $110 \pm 3$ & $123 \pm 2$ \\
\hline 45 & $109 \pm 3$ & $120 \pm 5$ & $130 \pm 1$ \\
\hline 60 & $124 \pm 9$ & $136 \pm 1$ & $150 \pm 6$ \\
\hline Modelo & $\begin{array}{c}y=0,85 x+73 \\
R^{2}=0,993 * *\end{array}$ & $\begin{array}{c}y=0,83 x+85 \\
R^{2}=0,997 * *\end{array}$ & $\begin{array}{c}y=0,78 x+99 \\
R^{2}=0,974 * *\end{array}$ \\
\hline
\end{tabular}

$* \mathrm{e} * *$ significativo a 5 e $1 \%$ de probabilidade, respectivamente, pelo teste $\mathrm{F}$; ns = não significativo. 
ao desenvolvimento da cultura, ${ }^{38} \mathrm{o}$ curto tempo de crescimento das plantas que foram colhidas aos 40 dias (entre os estágios V4 e V5) e possíveis desordens nutricionais, fundamentalmente na maior das doses de LE, podem ter contribuído também para o baixo rendimento de matéria seca. Em geral, o pH no solo $<7,0$ diminui a absorção de cátions, talvez por competição entre estes e $\mathrm{H}^{+}$por sítios do carregador. $^{38}$

Tabela 3. Matéria seca, teor e acúmulo de $\mathrm{Zn}$ da parte aérea das plantas de milho, crescidas em solo de diferente $\mathrm{pH}$, tratado com doses de LE

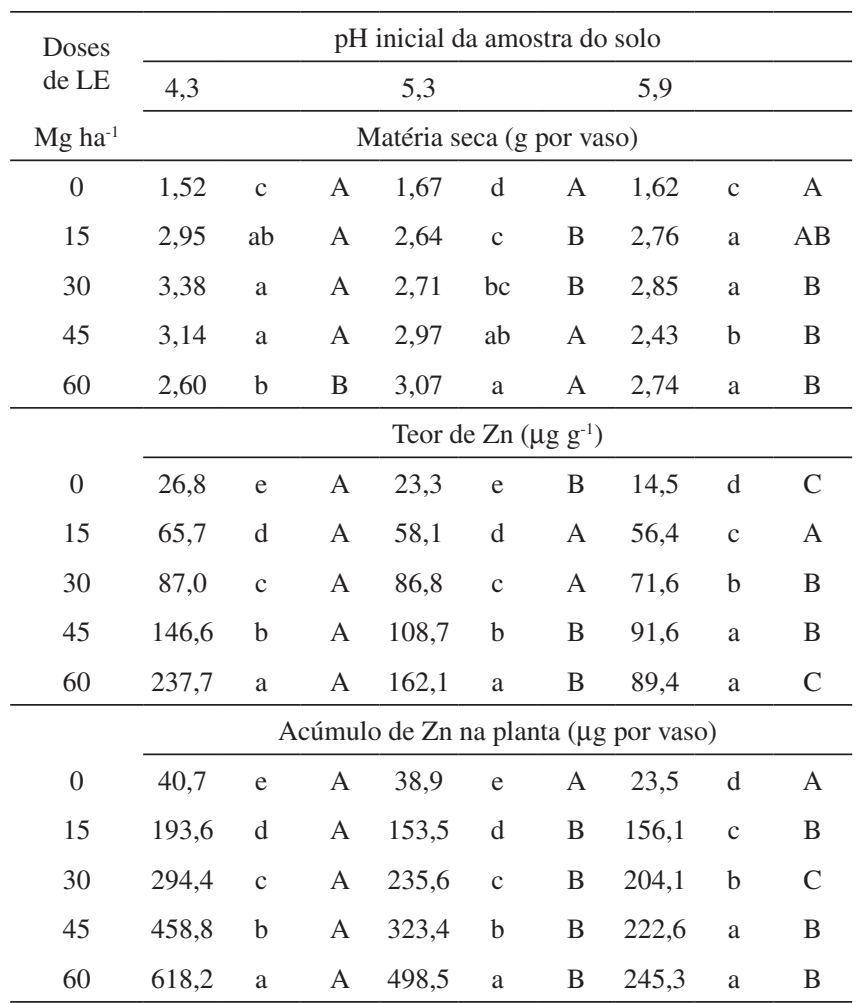

Médias com letras iguais, minúsculas nas colunas e maiúsculas nas linhas dentro de cada dose de LE, não diferem a 5\% pelo teste de Tukey.

O teor de Zn nas plantas aumentou com as doses de LE (Tabela 3). $\mathrm{O}$ maior teor de $\mathrm{Zn}$ na planta foi devido à dose de $60 \mathrm{Mg}$ ha $^{-1}$ de $\mathrm{LE}$, que promoveu aumentos de 8,8; 6,9 e 6,1 vezes, em relação ao teor encontrado no tratamento sem aplicação de LE, nas amostras de solo de $\mathrm{pH} 4,3 ; 5,3$ e 5,9, respectivamente. A quantidade de $\mathrm{Zn}$ adicionada ao solo via LE, 14,7; 29,4; 44,1 e 58,87 $\mathrm{mg} \mathrm{kg}^{-1}$, não alcançou o teor de prevenção para solos $\left(300 \mathrm{mg} \mathrm{kg}^{-1}\right) .{ }^{27} \mathrm{O}$ fato de o lodo ter aumentado a acidez do solo (Tabela 2) favoreceu o incremento da disponibilidade do elemento, como demonstram os resultados da fitodisponibilidade de $\mathrm{Zn}$ (valor $L$ ) (Tabela 4). O efeito da acidez sobre o teor de $\mathrm{Zn}$ na planta também foi observado em função do $\mathrm{pH}$ inicial das amostras de solo. $\mathrm{Na}$ amostra de solo com $\mathrm{pH}$ inicial 4,3, o teor de $\mathrm{Zn}$ foi maior e decresceu nas amostras de solo de pH 5,3 e 5,9 (Tabela 3) a partir da dose de $30 \mathrm{Mg} \mathrm{ha}^{-1}$. Nos tratamentos testemunha, o teor de $\mathrm{Zn}$ na parte aérea da planta esteve dentro da faixa adequada em folha ${ }^{39}$ (15 a $50 \mu \mathrm{g} \mathrm{g}^{-1}$ ) e superior a esta em todos os tratamentos com LE, chegando a ser ligeiramente superior ao limite inferior de toxidez $\left(200 \mu \mathrm{g} \mathrm{g}^{-1}\right)$ nas plantas, ${ }^{39}$ no tratamento com dose de $60 \mathrm{Mg} \mathrm{ha}^{-1} \mathrm{de}$ $\mathrm{LE}$, na amostra de solo com $\mathrm{pH}=4,3$.

$\mathrm{O} \mathrm{Zn}$ acumulado nas plantas de milho apresentou o mesmo comportamento que o teor de $\mathrm{Zn}$ e do rendimento de matéria seca, em função do pH do solo e das doses de LE, ou seja, dentro dos tratamentos de dose de LE, diminuiu nos tratamentos com amostra de solo de $\mathrm{pH}=4,3$ até a amostra de solo de $\mathrm{pH}=5,9$ e aumentou
Tabela 4. Valor $L$ de $\mathrm{Zn}$ no solo e coeficiente de regressão com as doses de LE, teor e acúmulo de $\mathrm{Zn}$ nas plantas de milho, crescidas em solos de diferentes $\mathrm{pH}$

\begin{tabular}{ccccccc}
\hline \multirow{2}{*}{ Doses de LE } & \multicolumn{5}{c}{$\mathrm{pH}$ inicial da amostra do solo } \\
\cline { 2 - 6 } Mg ha $^{-1}$ & \multicolumn{5}{c}{ 4,3,3 } & \multicolumn{5}{c}{5,9} \\
\cline { 2 - 6 } 0 & 1,4 & $\mathrm{~A}$ & 1,6 & $\mathrm{~A}$ & 1,4 & $\mathrm{~A}$ \\
15 & 10,8 & $\mathrm{~A}$ & 8,4 & $\mathrm{~A}$ & 10,3 & $\mathrm{~A}$ \\
30 & 15,0 & $\mathrm{~B}$ & 21,7 & $\mathrm{~A}$ & 20,2 & $\mathrm{~A}$ \\
45 & 24,8 & $\mathrm{C}$ & 29,5 & $\mathrm{~B}$ & 33,5 & $\mathrm{~A}$ \\
60 & 34,1 & $\mathrm{~B}$ & 45,1 & $\mathrm{~A}$ & 37,2 & $\mathrm{~B}$ \\
Modelo & $\mathrm{y}=0,52 \mathrm{x}+1,3$ & $\mathrm{y}=0,72 \mathrm{x}-0,3$ & $\mathrm{y}=0,63 \mathrm{x}+1,5$ \\
$\mathrm{R}^{2}$ & $0,987 * *$ & $0,982^{* *}$ & $0,980^{* *}$ \\
\hline \multicolumn{7}{c}{ Teor de Zn vs. Valor $L$ de Zn } \\
\hline Modelo & $\mathrm{y}=6,4 \mathrm{x}+2,6$ & $\mathrm{y}=3,0 \mathrm{x}+23,5$ & $\mathrm{y}=1,9 \mathrm{x}+24,3$ \\
$\mathrm{R}^{2}$ & $0,967 * *$ & $0,987 * *$ & $0,894 * *$ \\
\hline \multicolumn{7}{c}{ Acúmulo de Zn vs. Valor $L$ de Zn } \\
\hline Modelo & $\mathrm{y}=17,8 \mathrm{x}+14,4$ & $\mathrm{y}=10,0 \mathrm{x}+36,7$ & $\mathrm{y}=5,3 \mathrm{x}+61,1$ \\
$\mathrm{R}^{2}$ & $0,998^{* *}$ & $0,985^{* *}$ \\
\hline
\end{tabular}

Médias com letras iguais, nas linhas dentro de cada dose de LE, não diferem a 5\% pelo teste de Tukey. **Regressão significativa a $P \leq 0,01$ pelo teste $\mathrm{F}$.

com as doses de LE (Tabela 3). O incremento do acúmulo de Zn na planta, com a maior dose de LE, e em relação ao tratamento sem LE foi de 15,1; 12,8 e 10,4 vezes nas amostras de solo de $\mathrm{pH}=4,3 ; 5,3$ e 5,9, respectivamente. Em relação ao $\mathrm{Zn}$ total no solo ( $\mathrm{Zn}$ do solo mais $\mathrm{Zn}$ adicionado via $\mathrm{LE}$ ), a quantidade deste elemento absorvido pelas raízes das plantas representou entre 0,24 e $0,36 \%$ na amostra de solo de $\mathrm{pH}=4,3$, entre 0,19 e $0,29 \%$ na amostra de solo de $\mathrm{pH}=5,3$ e de 0,19 a $0,14 \%$ na amostra de solo com $\mathrm{pH}=5,9$. O pH do solo é o fator que tem maior influência sobre a disponibilidade de $\mathrm{Zn} \cdot{ }^{13,14,40} \mathrm{~A}$ elevação de uma unidade do $\mathrm{pH}$ no solo provoca uma diminuição de 100 vezes na concentração do $\mathrm{Zn}^{2+}$ da solução do solo. ${ }^{13} \mathrm{~A}$ elevação do pH no solo aumenta a adsorção e a fixação do $\mathrm{Zn}$ nas argilas e na $\mathrm{MO}$, assim como a formação de compostos de baixa solubilidade no solo $\left(\mathrm{ZnCO}_{3}\right.$ e $\left.\mathrm{ZnPO}_{4}\right)$. Simultaneamente, na planta há aumento da absorção de cátions ao reduzir a competição dos íons $\mathrm{H}^{+}$pelos mesmos sítios do carregador iônico. ${ }^{14,39}$

As substâncias húmicas contêm grande número de grupos funcionais $(-\mathrm{OH},-\mathrm{COOH},-\mathrm{SH}$ e $-\mathrm{C}=\mathrm{O})$ com afinidade para metais de transição, além de serem dependentes da qualidade dos constituintes orgânicos e pH do solo. Dessa forma, são formados tanto complexos solúveis como pouco solúveis, regulando a solubilidade e disponibilidade dos elementos na solução do solo para posterior absorção pelas raízes das plantas. ${ }^{36}$ Assim, componentes orgânicos de baixo peso molecular, por exemplo, ácidos fúlvicos, são carregadores de Zn na solução do solo, com constantes de estabilidade dependentes do $\mathrm{pH}$, enquanto componentes de alto peso molecular (ácidos húmicos) podem imobilizar o $\mathrm{Zn}$, sendo estes considerados reservatórios de Zn no solo. ${ }^{14,36}$ Porém, a fitodisponibilidade de Zn determinada pelo valor $L$ (Tabela 4 ) foi crescente com o aumento do $\mathrm{pH}$ no solo, aparentando uma contradição com o resultado interpretado para o teor e acúmulo de $\mathrm{Zn}$ nas plantas, uma vez que estes diminuíram com o pH do solo e o valor $L$ aumentou, quando comparado o efeito do valor de $\mathrm{pH}$ inicial dentro das doses de lodo, notadamente para 30,45 e $60 \mathrm{Mg} \mathrm{ha}^{-1}$.

Como o valor $L$ é calculado pela relação entre as atividades específicas do ${ }^{65} \mathrm{Zn}$ adicionado ao solo e a atividade específica na planta, de acordo com a Equação 2, o aumento do valor $L$ indica que houve 
diminuição da atividade específica do ${ }^{65} \mathrm{Zn}$ da planta com o aumento do $\mathrm{pH}$ do solo. Esse resultado pode ser explicado por ocorrência de diluição isotópica do ${ }^{65} \mathrm{Zn}$ com a fração do elemento disponível na solução do solo, ou por troca isotópica com a fração do $\mathrm{Zn}$ na fase sólida e em equilíbrio com a solução do solo. O antagonismo do $\mathrm{Zn}^{2+}$ com o $\mathrm{Ca}^{2+}$ pelos sítios de adsorção no solo, ${ }^{14}$ ou com o $\mathrm{Zn}$ complexado pela $\mathrm{MO}$, na medida em que o $\mathrm{pH}$ do solo aumentou, também pode ter favorecido a diluição ou troca isotópica. Por outro lado, as raízes das plantas de milho, quando em condições ideais de $\mathrm{pH}(\geq 5,5)$ para o seu desenvolvimento e absorção de nutrientes, ${ }^{38,41}$ poderiam ter absorvido $Z n$ de uma fração menos disponível, ou seja, aquela que não teve marcação do $\mathrm{Zn}$ por troca isotópica com o ${ }^{65} \mathrm{Zn}$ e que induziu a diluição isotópica, diminuindo assim a atividade específica do ${ }^{65} \mathrm{Zn}$ na planta e, consequentemente, aumentando o valor $L$. Pode-se afirmar, então, que a redução do teor e acúmulo de $\mathrm{Zn}$ nas plantas, dentro da dose de LE com o aumento do $\mathrm{pH}$ inicial do solo, mesmo que o valor $\mathrm{L}$ tenha aumentado, pode ter suas causas no antagonismo do $\mathrm{Zn}^{2+}$ com os íons de $\mathrm{Cu}^{2+}, \mathrm{Mn}^{2+} \mathrm{e} \mathrm{Ni}^{2+}$, devido à competição pelos mesmos sítios do carregador, como explicado por Havlin et al.. ${ }^{20}$

O teor de Zn fitodisponível, obtido pelo método isotópico do valor $L$, apresentou relação linear com as doses de LE adicionadas ao solo (Tabela 4). Considerando a menor (15 $\left.\mathrm{Mg} \mathrm{ha}^{-1}\right)$ e maior $\left(60 \mathrm{Mg} \mathrm{ha}^{-1}\right)$ dose de LE em relação ao tratamento sem lodo, houve aumentos de 7,7 a 24,3 vezes na fitodisponibilidade de $\mathrm{Zn}$ no solo com $\mathrm{pH}$ inicial de 4,3; de 5,2 a 28,1 vezes no solo com $\mathrm{pH}$ inicial de 5,3 e de 7,4 a 26,8 vezes no solo com $\mathrm{pH}$ inicial de 5,9.

Houve relações lineares entre o teor de $\mathrm{Zn}$ e o acumulado na planta com o valor $L$, nos três níveis de $\mathrm{pH}$ do solo (Tabela 4). Os altos coeficientes de regressão, todos com $1 \%$ de significância, indicaram que a metodologia isotópica foi eficiente na avaliação da fitodisponibilidade de Zn para a cultura do milho, nas condições experimentais de solo tratado com LE. Isto demonstra que o método isotópico do valor $L$ é função integrada dos fatores químico-físicos do solo, que definem a disponibilidade do $\mathrm{Zn}$, e os fatores químico-físico-fisiológicos da planta, que regulam os processos de absorção de Zn pelas raízes.

Observou-se que o coeficiente angular da regressão, entre o teor e acúmulo de Zn nas plantas de milho e o valor $L$, diminuiu de 6,40 para 1,96 e de 17,81 para 5,30, nas amostras de solo com valor de $\mathrm{pH}=4,3$ até 5,9 , respectivamente. Portanto, para cada $\mathrm{mg}$ de $\mathrm{Zn}$ fitodisponível (Valor $L$ ) por $\mathrm{kg}$ do solo, houve um decréscimo de $30 \%$ do elemento no tecido das plantas com o aumento do $\mathrm{pH}$ do solo (Tabela 4).

A porcentagem de Zn fitodisponível no solo, calculada com base no valor $L$ em relação à quantidade de $\mathrm{Zn}$ total presente no solo ( $\mathrm{Zn}$ do solo $+\mathrm{Zn}$ do LE), aumentou com as doses de LE e com o pH do solo, sendo maior nos tratamentos com LE do que na testemunha sem aplicação de lodo (Tabela 5). Estes valores indicam que tanto o solo como o próprio LE atuaram como fonte e dreno do $\mathrm{Zn}$. As plantas mostraram baixas porcentagens de utilização do $\mathrm{Zn}$ fitodisponível, com valores decrescentes com o aumento do $\mathrm{pH}$ inicial do solo (Tabela 5) e valores decrescentes com o aumento da dose de LE, o que confirma o papel regulador das condições químicas e químico-físicas do solo sobre os processos fisiológicos da planta na absorção do Zn.

As porcentagens de Zn do solo (\% Znpps) e do LE (\% ZnppLE) nas plantas (Tabela 6) foram calculadas pelas Equações 3 e 4 , respectivamente. Observa-se que houve aumento na absorção de $\mathrm{Zn}$ do lodo pelas raízes das plantas, enquanto que o $\mathrm{Zn}$ absorvido do solo diminuiu com as doses de LE. Esse resultado foi devido à adição de Zn e à acidificação do solo pelas doses de LE (Tabela 2), porém, dentro de cada dose de LE não foi observado efeito do $\mathrm{pH}$ do solo sobre a \% Znpps e a \% ZnppLE.
Tabela 5. Porcentagem de $\mathrm{Zn}$ fitodisponível e de sua utilização pelas plantas crescidas em solos de diferentes $\mathrm{pH}$ tratados com doses de lodo

\begin{tabular}{cccccc}
\hline \multirow{2}{*}{$\mathrm{pH}$} & \multicolumn{5}{c}{ Doses de LE $\left(\mathrm{Mg} \mathrm{ha}^{-1}\right)$} \\
\cline { 2 - 5 } & 0 & 15 & 30 & 45 & 60 \\
\hline \multirow{5}{*}{ \% Zn fitodisponível no solo } \\
\cline { 2 - 6 } 5,3 & 5,5 & 26,4 & 26,9 & 35,3 & 40,1 \\
5,9 & 6,3 & 20,5 & 39,0 & 42,0 & 53,1 \\
\hline & 5,4 & 25,3 & 36,5 & 47,8 & 43,8 \\
\hline 4,3 & 1,42 & 0,90 & 0,99 & 0,93 & 0,91 \\
5,3 & 1,18 & 0,92 & 0,54 & 0,55 & 0,56 \\
5,9 & 0,84 & 0,75 & 0,50 & 0,33 & 0,33 \\
\hline
\end{tabular}

Tabela 6. Porcentagem de Zn do solo absorvido pelas plantas (\% Znpps) e do LE (\% ZnppLE), em função das doses de LE e pH do solo

\begin{tabular}{cccccc}
\hline \multirow{2}{*}{$\mathrm{pH}$} & $\%$ & \multicolumn{4}{c}{ Doses de LE $\left(\mathrm{Mg} \mathrm{ha}^{-1}\right)$} \\
\cline { 3 - 6 } & & 15 & 30 & 45 & 60 \\
\hline \multirow{2}{*}{4,3} & Znpps & $13 \pm 1$ & $9 \pm 1$ & $5,6 \pm 0,5$ & $5,1 \pm 0,2$ \\
& ZnppLE & $87 \pm 1$ & $91 \pm 1$ & $94,4 \pm 0,5$ & $94,9 \pm 0,2$ \\
\multirow{2}{*}{5,3} & Znpps & $20 \pm 1$ & $8 \pm 1$ & $5,6 \pm 0,2$ & $3,7 \pm 0,2$ \\
& ZnppLE & $80 \pm 1$ & $92 \pm 1$ & $94,4 \pm 0,2$ & $96,3 \pm 0,2$ \\
\multirow{2}{*}{5,9} & Znpps & $14 \pm 1$ & $7 \pm 1$ & $4,1 \pm 0,1$ & $3,9 \pm 0,3$ \\
& ZnppLE & $86 \pm 1$ & $93 \pm 1$ & $95,9 \pm 0,1$ & $96,1 \pm 0,3$ \\
\hline
\end{tabular}

\section{CONCLUSÃO}

As doses de lodo de esgoto promoveram aumentos da acidez ativa e potencial do solo, dos teores de $\mathrm{MO}, \mathrm{Ca}, \mathrm{Mg}$, da capacidade de troca de cátions, do teor de $\mathrm{Zn}$ no solo e da fitodisponibilidade (valor $L$ ) de $\mathrm{Zn}$. O incremento do teor de $\mathrm{Zn}$ no solo, na maior dose de lodo aplicada, não chegou a alcançar o valor de prevenção para solo.

A diminuição do $\mathrm{pH}$ do solo com uso de lodo ácido pode, conforme a dose aplicada, alcançar valores de acidez prejudicial para o desenvolvimento das culturas, indicando a necessidade de se realizar a calagem do solo depois da aplicação do lodo.

As correlações lineares significativas do Zn na planta (concentração e total) permitiram qualificar o método isotópico do valor $L$ como bom estimador da fitodisponibilidade de $\mathrm{Zn}$ em solo de diferentes $\mathrm{pH}$ tratado com lodo de esgoto. Assim mesmo, o método isotópico permitiu discriminar na planta a origem do $\mathrm{Zn}$ absorvido (se do solo ou do lodo) e estimar que a porcentagem de Zn, derivado do LE nas plantas (\% ZnppLE) aumentou com a dose de lodo, enquanto que a porcentagem de $\mathrm{Zn}$ proveniente do solo (\% Znpps) diminuiu.

\section{AGRADECIMENTOS}

À FAPESP (Fundação de Amparo à Pesquisa do Estado de São Paulo) pelo apoio financeiro e ao Centro de Energia Nuclear na Agricultura, da USP, pelo apoio logístico e disponibilização da infraestrutura laboratorial.

\section{REFERÊNCIAS}

1. Abreu Junior, C. H.; Boaretto, A. E.; Muraoka, T.; Kiehl, J. C.; Tópicos Ciên. Solo 2005, 4, 391.

2. Franco, A.; Abreu Junior, C. H.; Perecin, D.; Oliveira, F. C.; Granja, A. 
C. R.; Braga, V. S.; Rev. Bras. Ciên. Solo 2010, 34, 553; Gomes, S. B. V.; Nascimento, C. W. A.; Biondi, C. M.; Rev. Bras. Eng. Agríc. Ambiental 2007, 11, 459; Rathod, P. H.; Patel J. C.; Jhala, A. J.; Commun. Soil Sci. Plant Anal. 2011, 42, 263.

3. Tsutiya, M. T. Em Biossólido na agricultura; Tsutiya, M. T.; Comparini, J. B.; Alem Sobrinho, P., eds.; ABES/SP: São Paulo, 2002, cap. 4.

4. Page, A. L.; Geason, T. L.; Smith, J. E.; Iskandar, J. K.; Sommers, L. E., eds.; Utilization of municipal wastewater and sludge on land, University of California: Riverside, 1983.

5. Valadares, J. M. A. S.; Gal, M.; Milgelgrin, V.; Page, A. L.; J. Environ. Qual. 1983, 12, 49.

6. Bergmann, W.; Nutritional disorders of plants: development, visual and analytical diagnosis, Jena: Stuttgart; G. Fischer: New York, 1992.

7. Epstein, E.; Bloom, A. J.; Mineral nutrition of plants: Principles and perspective, $2^{\text {nd }}$ ed., Sinauer Associates: Sunderland, 2005.

8. Brasil, Conselho Nacional do Meio Ambiente; Resolução $N^{o} 375$, de 29/8/2006.

9. Oliveira, F. C.; Mattiazzo, M. E.; Sci. Agric. 2001, 58, 581; Macdonald, C. A.; Clark, I. M.; Zhao, F.; Hirsch, P. R.; Singh, B. K.; McGrath, S. P.; Soil Biol. Biochem. 2011, 43, 932.

10. Chaney, R. L. Em Zinc in Soils and Plants; Robson, A. D., ed.; Kluwer Academic Publishers: Dordrecht, 1993, cap. 10.

11. Carpentier, R. Em Handbook of Plant and Crop Physiology; Pessarakli, M., ed.; $2^{\text {nd }}$ ed., Marcel Dekker Inc.: New York, 2002, cap. VII.

12. Sandstead, H. H.; AU, W. Em Handbook on the toxicology of metals; Nordberg, G. F.; Fowler, B. A.; Nordberg, M.; Friberg, L., eds.; $3^{\text {rd }}$ ed., Academic Press, Elsevier Inc.: California, 2007, cap. 47.

13. Kabata-Pendias, A.; Pendias, H.; Trace Elements in Soils and Plants, $4^{\text {th }}$ ed., CRC Press: Boca Raton, 2010.

14. Alloway, B. J.; Heavy Metals in Soils, $2^{\text {nd }}$ ed., Blackie Academic \& Professional: London, 1995.

15. Reis, T. C.; Rodella, A. A.; Rev. Bras. Ciên. Solo 2002, 26, 619; Pereira, M. G.; Pérez, D. V.; Valladares, G. S.; Souza, J. M. P. F.; Anjos, L. H. C.; Rev. Bras. Ciên. Solo 2001, 25, 655; Leea, S.; Park, H.; Koo, N.; Hyun, S.; Hwang, A.; J. Hazard. Mater. 2011, 188, 44.

16. Tessier, A.; Campbell, P. G. C.; Bisson, M.; Anal. Chem. 1979, 51, 844; Buccolieri, A.; Buccolieri, G.; Dell'Atti, A.; Strisciullo, A.; GaglianoCandela, R.; Environ. Monit. Assess. 2010, 168, 547.

17. Berton, R. S.; Valadares, J. M. A. S.; Camargo, O. A. de; Bataglia, O. C.; Rev. Bras. Ciênc. Solo 1997, 21, 685.

18. Hooda, P. S.; Alloway, B. J.; Sci. Total Environ. 1994, 149, 39.

19. Oliveira, F. C.; Mattiazzo, M. E.; Marciano, C. R.; Rosseto, R.; Rev. Bras. Ciên. Solo 2002, 26, 505.

20. Havlin, J. L.; Beaton, J. D.; Tisdale, S. L.; Nelson, W. L.; Soil fertility and fertilizers: An introduction to nutrient management, $7^{\text {th }}$ ed.; Prentice Hall: New Jersey, 2005.

21. Larsen, S.; Plant Soil 1952, 4, 1.

22. Alvarez, V. F. C.; Boaretto, A. E.; Abreu Jr., C. H.; Muraoka, T.; Trevizam, A. R.; Nascimento-Filho, V. F.; Quim. Nova 2008, 31, 1020; Alvarez, V. F. C.; Boaretto, A. E.; Abreu Jr., C. H.; Muraoka, T.; Trevizam, A. R.; Fernandes, H. M. G.; Quim. Nova 2008, 31, 990; International Atomic Energy Agency; Use of Isotope and Radiation Methods in Soil and Water Management and Crop Nutrition, Training Course Series 14, IAEA: Vienna, 2001; Hettiarachchi, G. M.; Lombi, E.; McLaughlin, M. J.; Chittleborough, D. J.; Johnston, C.; Aust. J. Soil
Res. 2010, 48, 238; Nolan, A. L.; Zhang, H.; McLaughlin, M. J.; J. Environ. Qual. 2005. 34, 496; Wu, C.; Lu, L.; Yang, X.; Feng, Y.; Wei, Y.; Hao, H.; Stoffella, P. J.; He, Z.; J. Agric. Food Chem. 2010, 58, 6767; Sivry, Y.; Riotte, J.; Sappin-Didier, V.; Munoz, M.; Redon, P.; Denaix, L.; Dupré, B.; Environ. Sci. Technol. 2011, 45, 6247.

23. FAO; World reference base for soil resources; World soil resources reports $N^{\circ}$ 103, Rome, 2006.

24. Camargo, O. A.; Moniz, A. C.; Jorge, J. A.; Valadares, J. M. A. S.; Métodos de análise química, mineralógica e física de solos do Instituto Agronômico de Campinas, IAC: Campinas, 1986 (Boletim Técnico, 106).

25. van Raij, B.; Quaggio, J. A.; Andrade, J. C.; Cantarella, H.; Quaggio, J. A.; Análise química para avaliação da fertilidade de solos tropicais, IAC: Campinas, 2001.

26. U.S. EPA - United State Environment Protection Agency; SW-846, Test Methods for Evaluating Solid Waste, Physical Chemical Methods, 2007.

27. Brasil, Conselho Nacional do Meio Ambiente; Resolução $N^{o} 420$, de 28/12/2009.

28. Nelson, D. W.; Sommers, L. E. Em Methods of soil analysis. Part 3, Chemical methods; Bigham, J. M., ed.; Soil Science Society of America, Inc., American Society of Agronomy Inc.: Madison, 1996, cap. 34.

29. van Raij, B.; Cantarella, H.; Quaggio, J. A.; Furlani, A. M. C.; Recomendações de adubação e calagem para o Estado de São Paulo, $2^{\mathrm{a}}$ ed., Instituto Agronômico de Campinas: Campinas, 2001.

30. Bataglia, O. C.; Furlani, A. M. C.; Teixeira, J. P. F.; Furlani, P. R.; Gallo, J. R.; Métodos de análise química de plantas, Instituto Agronômico de Campinas: Campinas, 1983.

31. Thompson, M.; Ellison, S. L. R.; Wood, R.; Pure Appl. Chem. 2002, 74 , 835.

32. Instituto Nacional de Metrologia, Normalização e Qualidade Industrial; DOQ-CGCRE-008, Orientações sobre Validação de Métodos de Ensaios Químicos, Março, 2003.

33. SAS Institute; SAS system for Windows. Release 8.02, SAS Institute: Cary, NC., 2001.

34. Nascimento, C. W. A.; Barros, D. A. S.; Melo, E. E. C.; Oliveira, A. B.; Rev. Bras. Ciên. Solo 2004, 28, 385.

35. Bovi, M. L. A.; Godoy, G.; Da Costa, E. A. D.; Berton, R. S.; Spiering, S. H.; Veja, F. V. A.; Cembranelli, M. A. R.; Maldonado, C. A. B.; Rev. Bras. Ciên. Solo 2007, 31, 153

36. Chen, Y.; Avnimelech, Y., eds.; The role of organic matter in modern agriculture, Martinus Nijhoff: Dordrecht, 1986; Chen, Y. Em Humic substances in terrestrial ecosystems; Piccolo, A., ed.; Elsevier: Amsterdam, 1996; Kamali, S.; Ronaghi, A.; Karimian, N.; Commun. Soil Sci. Plant Anal. 2011, 42, 1038.

37. Simonete, M. A.; Kiehl, J. C.; Sci. Agric. 2002, 59, 555.

38. Malavolta, E.; Elementos de nutrição mineral de plantas, Agronômica Ceres: São Paulo, 1980.

39. Malavolta, E.; Fertilizantes e seu impacto ambiental. Micronutrientes e metais pesados, mitos, mistificação e fatos, Produquímica: Piracicaba, 1994.

40. Lindsay, W. L. Em Micronutrients in Agriculture; Morvtvedt, J. J.; Giordano, P. M.; Lindsay, W. L., eds.; Soil Sci. Soc. America, Inc.: Madison, 1972 .

41. Marschner, H.; Mineral nutrition of higher plants, Academic Press: Orlando, 1986. 